concussion (23.3\%), followed by injury to the wrist/hand $(22.7 \%)$, shoulder $(16.7 \%)$, and knee $(14.3 \%)$. Over a half $(52.1 \%)$ of injury events occurred on downhills. Males and females reported similar injury rates $(2.43 \%$ and $2.86 \%$, respectively), but females sustained significantly more lower limb injuries $(p=0.003)$. A large portion $(72.4 \%)$ of injured athletes were unable to complete the ride; $49.3 \%$ of crashes resulted in an emergency room visit.

Conclusions Acute traumatic injuries are common in mountain bike racing, including concussions and injuries to the upper extremities. Males and females have similar injury rates but different injury types. To our knowledge, this is the largest mountain bike racing ISS in existence.

\section{INJURIES AMONG YOUTH MOUNTAIN BIKE RACING COACHES: THREE-YEAR DATA FROM A NATION-WIDE INJURY SURVEILLANCE SYSTEM IN THE UNITED STATES}

${ }^{1}$ Meredith Ehn, ${ }^{1}$ Daniel Cushman, ${ }^{1}$ Masaru Teramoto, ${ }^{2}$ Chris Spencer, 'Stuart Willick. ${ }^{1}$ University of Utah, Salt Lake City, USA; ${ }^{2}$ National Interscholastic Cycling Association, Berkeley, USA

\subsection{6/bjsports-2021-IOC.336}

Background As youth mountain bike racing is a rapidly growing sport in the United States, it is essential to monitor injuries in both student-athletes and coaches who ride with student-athletes during practices. An injury surveillance system (ISS) implemented through a collaboration between University of Utah researchers and the National Interscholastic Cycling Association (NICA), referred to as NICA-ISS, helps to better understand injury characteristics in this sport and pursue datadriven injury prevention strategies.

Objective Describe the types of and factors associated with injuries sustained by coaches during NICA-sanctioned mountain bike training.

Design Data were collected via NICA-ISS, developed using a web-based system, from NICA leagues during the 2018-2020 racing seasons. A designated reporter from each team reported injuries sustained by coaches along with those sustained by student-athletes.

Setting Nation-wide youth mountain biking leagues in the United States.

Participants NICA coaches participating in the 2018-2020 seasons. Assessment of Risk Factors Variables analyzed included injury characteristics, coach demographics, and factors associated with injuries.

Main Outcome Measurements Summary measures (frequency and proportion) of injuries and associated factors.

Results Injuries were tracked in 31,280 coach-years, resulting in 407 injuries in 227 reported injury events. The most commonly reported injury was to the shoulder (34.8\%), followed by concussion (17.6\%) and wrist/hand (17.6\%). $57.3 \%$ of injuries occurred on downhills. Males and females reported similar injury rates $(0.70 \%$ and $0.80 \%$, respectively). Injury characteristics differed between sexes, with female coaches sustaining more lower limb injuries while males sustained more upper limb injuries. Whereas $56.4 \%$ of crashes resulted in an emergency room visit, $9.3 \%$ required hospital admission.
Nearly half $(48.3 \%)$ of injury events resulted in time-loss of 4 weeks or greater.

Conclusions Acute traumatic injuries are common in mountain bike racing coaches and in student-athletes. Dissimilarly to student-athletes, coaches sustain upper extremity injuries more often than concussions. To our knowledge, this is the first ISS to track injuries in mountain bike racing coaches.

\section{SPORTS-RELATED CONCUSSION (SRC) IN ROAD CYCLING: ESTABLISHING THE ROADSIDE HEAD INJURY ASSESSMENT (RIDE) FOR ELITE ROAD CYCLING}

${ }^{1,2}$ Neil Heron, ${ }^{2}$ Elliott Jonathan. ${ }^{1}$ Keele University, Keele, UK; ${ }^{2}$ Queen's University Belfast, Belfast, UK

\subsection{6/bjsports-2021-IOC.337}

Background Despite recent advances in the diagnosis and management of sports-related concussion (SRC) in the sports medicine community; as well as heightened recognition of the condition by the public and media, some sports, such as road cycling, appear to lack effective concussion assessment, diagnosis and management protocols.

Objectives Undertake a systematic review of the literature on SRC assessment in road cycling and from this evidence, suggest a model for the Roadsile heaD injury assessment (RIDE) as well model return to riding guidelines.

Design Systematic review.

Setting Elite Sport.

Patients (Or Participants) This systematic review is reported in line with the Preferred Reporting Items for Systematic Reviews and Meta-analyses guidance.

Interventions (Or Assessment Of Risk Factors) The Physiotherapy Evidence Database (PEDro) scale was used to assess the quality of included papers in the review.

Main Outcome Measurements From 94 studies identified, 65 studies were excluded after screening the titles and abstracts and two studies were included in the review.

Results Gordon et al describe the presentation of a single case of paediatric concussion following a cycling crash. They highlight the utility of SRC evaluation using the Sport Concussion Assessment Tool (SCAT) as well as the importance of a stepwise return-to-play protocol. Greve and Modabber discuss a number of traumatic brain injuries that occurred during the 2011 road cycling season and,as a minimum, call for riders to be withdrawn from competition following loss of consciousness or amnesia. From this review, we then suggest a Roadside head injury assessment (RIDE) for assessing SRC in elite road cycling.

Conclusions The elite road cycling race environment poses a unique challenge to the identification of suspected SRC and this review illustrates the lack of published evidence to advise effective means of SRC assessment within road cycling. We are calling for The Union Cycliste Internationale (UCI) to host a consensus meeting to agree the operational detail required to implement a standardised RIDE - informed by the Berlin Consensus Guidelines, SCAT5 and return-to-riding protocol for road cycling. 